\section{Research Square}

\title{
Visual Reaction Time: A Tool for Reducing Road Traffic Collisions and Driving Simulator.
}

Prashant Rajdeep ( $\sim$ prashant.rajdeep-physiology@msubaroda.ac.in )

Baroda Medical College https://orcid.org/0000-0002-9047-8803

\section{Lajja Patel}

Baroda Medical College

\section{Steffy CD}

Baroda Medical College

\section{Preeti Panchal}

Baroda Medical College

\section{Original Contribution}

Keywords: Collision prevention, Reaction time, Cognition, Web, Driver classification, Simulation

Posted Date: November 30th, 2021

DOI: https://doi.org/10.21203/rs.3.rs-1063762/v1

License: @ (i) This work is licensed under a Creative Commons Attribution 4.0 International License. Read Full License 


\section{Abstract}

Objective-Attenuating post lockdown vehicular speed by employing visual reaction time as a tool to prime the citizens for creating decorum of driving and checking the road traffic fatalities.

Background- It is indispensable to curb the driving speed post lockdown to avoid accidents. Even though, the impact of inactivity on RT has been well established, an insight into the new method can deal with the gross issue of road traffic casualty worldwide.

Method- Using a web-based platform (http://physicsiology.com), quantification of post lockdown speed was achieved for 643 participants under average speed before lockdown and RT measurement.

Results-Compared to pre lockdown vehicular speed, reduced post lockdown speed was well calculated and suggested. Also, there was a correlation between RT, age, and days of lockdown.

Conclusions-Containment of speed can be achieved to prime people through RT. Additionally, RT can determine the rate of change of frequency (ROCOF) for detecting the swiftness of action (i.e., the brain's ability to deal with the transition between reaction times of different events) required for averting road traffic collisions. Compelling to suggest a need for a humanoid simulator that can garner real-time data.

Application-Suggesting a fresh outlook for designing a contraption for a better appraisal of the fleet in driving skills, thus beaconing the course towards restraining road traffic fatalities

\section{Background}

As India leaps towards becoming a developed nation, roadways play a crucial role in development, helping people reach far-off places. India has more than 210 million registered motor vehicles as of 2015, providing ease and comfort in daily commute (W.H.O 2018). But the luxury of having any means of commutation comes with a social responsibility of driving safely and sensibly. Driving is a skilled task that involves a complex interrelation of the brain's motor and cognitive functions and is acquired and stored as working memory with due practice (Rostami and Ashayeri 2009). The key factor in driving is speed; hence controlling the vehicle speed can mitigate collisions. Every year worldwide, around 1.35 million deaths occur due to road accidents (W.H.O 2018). This is a major part that can logically be attributed to breaking the traffic rules and regulations. In the wake of an emergency like the lockdown, the vehicles' speed can be unforeseeable, leading to chaos. Lockdown is a rare phenomenon. However, when it occurs, many drivers may lose the accustomed driving practice. Besides, many drivers drive essentially every day on roads without traffic jams, making them get used to driving at high speeds. Post lockdown, the traffic on roads would be a conflicting image of the one before the lockdown, as people have developed diverse capacities to drive during the lockdown, creating more chaos. An ideal way to prevent such mishaps is by formulating newer means to educate people on regulating vehicle speeds to avoid collisions in such incidences. 
Our intervention that effectively manages and controls a vehicle's speed is based on the principle of 'reaction time.' 'Visual reaction time' is simple, non-invasive physiological parameters that give information about the sensory and motor association (Collins and Long 1996). The exact distance at which a vehicle brakes to prevent a collision is based upon visual reaction time, providing a definite speed to drive at (Li and Chen 2017; Olson and Sivak 1986). We suggest that more research is required to explore the theme's expanse, aiding the administration in facilitating the road mishaps.

\section{Methods}

The study was based on an online survey due to the covid19 restrictions that forbid us to get hold of realtime driving test data, yielding accurate results to check the effect of inactivity on RT, which affects driving. With approval by ethical board of institute, a well-outlined primitive plan was laid in action to incorporate a large number of populations, motivating them to check their post lockdown speed. As a result, an interactive website, 'http://physicsiology.com,' took its form. The website was conceptualized by authors and developed by 'kinetic dreamers.' The participants in the present study were the general population across the country. The study was accomplished within 25 days of its inception on April 132020 , by sharing the link on the various social media platforms through self-promotion. The website consisted of a form with a short description of the study. The parameters obtained from the form used for analysis were age, country, days of lockdown, and average speed before lockdown.

\subsection{Theory/Calculation}

Upon filling up the onscreen form, one was directed to the visual stimulus test page carrying specific instructions. The appearance of a red square or a circle on the screen initiated the test, which was to be clicked with agility until the color turns green on termination of the test. It took twenty-four clicks to complete the test, and the result was depended upon the rate at which the participants responded to the stimuli. In the end, the participants had to submit the test, which was followed by a display of their aSRT (Average shortest reaction time), aLRT (Average longest reaction time), and tentative post lockdown speed. The middle twenty clicks' data were the only ones that were considered for computing post lockdown speed.

Quantification of post lockdown speed was done under reaction time obtained by these twenty clicks that were sorted in increasing order. The first ten reaction time were averaged for the shortest reaction time (SRT), and the last ten responses were averaged for the longest reaction time (LRT). The website calculates the average post lockdown speed using the basic formula for constant speed as given below in Eq. (1)

I.e., Average Speed After Lock Down (ASALD) = [Average Comfortable Speed (ACS) before lockdown X Average Shortest Reaction Time (aSRT)]/Average Largest Reaction Time (aLRT) Eq. (1)

The post lockdown speed thus obtained was used as a tool to counsel the participants for a speed check. 


\section{Results}

The data collected from 643 Indian participants were analyzed. Since the distribution of data was not normal, we have applied a non-parametric test. The test showed that the participants' median age was 32 years, whereas the median days of lockdown were 21 days. The median post lockdown speed computed using the proposed formula was $7.69 \mathrm{~m} \mathrm{~s}^{-1}\left(27.7 \mathrm{~km} \mathrm{~h}^{-1}\right)$, while the average reduction in Post Lockdown Speed was $4.80 \mathrm{~m} \mathrm{~s}^{-1}\left(17.3 \mathrm{~km} \mathrm{~h}^{-1}\right)$. While response time can be figured out using table 1 .

Table 1. Response time data.

\begin{tabular}{|lll|}
\hline & In seconds & IQR(Inter Quartile Range) \\
\hline Median SRT & 0.45 & 0.41 to 0.52 \\
\hline Median LRT & 0.7 & 0.58 to 1.00 \\
\hline
\end{tabular}

Upon further analysis using the Pearson's coefficient of correlation, the most striking result from the data showed correlation between age and aSRT $(r=0.24,95 \% \mathrm{Cl} 0.16$ to $0.31, p<0.0001)$. Similar affirmations were seen in the study by Woods et al. (Woods et al. 2015), where an increase in latencies was seen with the increase in age. However, the correlation of age with aLRT was non-significant. We see quite a different correlation between the duration of lockdown and aSRT and aLRT, as shown in table 2 .

Table 2. Correlation Between Duration of Lockdown and average SRT \& LRT.

\begin{tabular}{|llll|}
\hline Correlation between duration of lock down and aSRT & $r=-0.11$ & $p=0.007$ & $95 \% \mathrm{Cl}(-0.18$ to -0.03$)$ \\
\hline Correlation between duration of lock down and aLRT & $r=-0.04$ & $p=0.27$ & $95 \% \mathrm{Cl}(-0.12$ to 0.03$)$ \\
\hline
\end{tabular}

\section{Discussion}

Our expertise in navigating traffic makes us judge the vehicular speeds and distance between our vehicle and the vehicle ahead of us. Based on the assembly of substantial evidence stating that different brain areas work to regulate the speed while driving (Calhoun et al. 2002; Sakai et al. 2018), we derived the tentative post lockdown speed using the averaged visual RT and pre lockdown speed. Presenting our perspective concerning the variance in visual RT, which is the brain's time to process the information that involves sequences of events before the action is implemented, it could be one of the influencers for speed control.

It has been authenticated that RT increases with increasing age and the increase in inactivity duration (Woods et al. 2015). The disparity in our results, showing a negative correlation between the RT and duration of inactivity, implies the need for further investigation down the line. The plausible cause of this negative correlation is that due to lockdown, the participants' screen time with mobile, in particular, 
would have increased, which would have resulted in exceptional responses, as demonstrated by J. Huang et al. (Huang et al. 2017). However, the converse can also be true, which is akin to our assumption that inactivity can prolong the RT. Therefore, the negative correlation strengthens our hypothesis that RT increases with an increase in inactivity duration. Contrary to the expectation, we did not find a significant correlation between aLRT with age $(r=0.04, p=0.34,95 \% \mathrm{Cl}-0.04$ to 0.11$)$ and aLRT with days of lockdown (as in Table 2). The most likely reason for it could be because the shortest RT is a curiosity-driven initial response while the longest RT is the wearied one.

Due to pre- lockdown data unavailability, we consider SRT as an average RT before lockdown and LRT as an average RT after lockdown. Have a better understanding with an example- Before lockdown, a person $\mathrm{X}$ is riding with a constant ACS of $11.11 \mathrm{~m} \mathrm{~s}^{-1}\left(40 \mathrm{~km} \mathrm{~h}^{-1}\right)$ and at an average RT, which would be equal to $\mathrm{SRT}=0.5 \mathrm{~s}$. After lockdown, the same person has an average RT equal to LRT $=0.7 \mathrm{~s}$. Here we can have two alternatives -

\section{I-Constant Speed (no acceleration)}

For a rider riding at a constant speed (no acceleration). Distance travelled in time $(\mathrm{SRT})=11.11 \times 0.5=$ $5.555 \mathrm{~m}$, we call it as safety distance. Now for the brain to cruise post lockdown with a reduced RT of 0.7 s. The brain can tackle it in either of the two ways:

To maintain the same safe distance $(5.555 \mathrm{~m})$. The rider should drive at a constant speed of $=5.555 / 0.7=7.9357 \mathrm{~m} \mathrm{~s}^{-1}\left(28.5685 \mathrm{~km} \mathrm{~h}^{-1}\right)$

Or

To maintain the same constant speed $\left(11.11 \mathrm{~m} \mathrm{~s}^{-1}\right)$. The rider should maintain a safe distance of $=11.11 \times 0.7=7.777 \mathrm{~m}$

Knowing the traffic movement pattern, the brain is conditioned to maintain a safe distance and comfortable speed to avoid fatalities. It is unjust to ascribe only the RT for braking while omitting deceleration, as drivers use both back and forth. Hence,

\section{II-The Application Of The Brake.}

For rider applying brake (deceleration) and with an average rider max deceleration $=4.6 \mathrm{~m} \mathrm{~s}^{-2}$ (Zhu et al. 2013). (Even if we consider the negative correlation of RT with the days of lockdown in the present study, the post lockdown deceleration would still be higher than normal; hence contemplating driver maximum would be more appropriate). Distance travelled in time $(\mathrm{SRT})=11.11 \times 0.5-0.5 \times 4.6 \times(0.5)^{2}=4.98 \mathrm{~m}$ of 
minimum braking distance or safe distance. Similarly, for the brain to cruise post lockdown with a reduced $\mathrm{RT}$ of $0.7 \mathrm{~s}$. The brain can tackle it in either of the two ways:

To maintain the same safe distance $(4.98 \mathrm{~m})$. The rider should attain a speed of $=(4.98+0.5 \times 4.6 \times 0.7 \times 0.7) / 0.7=8.724 \mathrm{~m} \mathrm{~s}^{-1}\left(31.4064 \mathrm{~km} \mathrm{~h}^{-1}\right)$.

To maintain the same speed $\left(11.11 \mathrm{~m} \mathrm{~s}^{-1}\right)$. The rider should maintain a distance.

$=11.11 \times 0.7-(0.5 \times 4.6 \times 0.7 \times 0.7)=6.65 \mathrm{~m}$

Nevertheless, based on driving skill, condition of the road, and type of the vehicle, every individual has its range of deceleration, hindering the generalized application of average rider max in countries with poorly regulated traffic (Bokare and Maurya 2017). For analogous reasons, it was not implemented in the present study as well. Also, it may remain unchanged post lockdown. The calculations given above can be well acknowledged, but with the consideration that acceleration/deceleration cannot be taken into account due to lack of feasibility. Maintaining a safe distance at the lowest speed would be the safest approach, coexisting with uniform speed calculation. Thereupon we proceed with emphasizing visual RT and maintaining uniform speed. Therefore, a driver can use either a comfortable speed or safe distance, both conditioned in the brain, to be kept at a constant range to be used simultaneously subconsciously while driving. This speed is achieved in the best possible way through fine-tuning the RT and acceleration or deceleration. This learned process often gives a sense of euphoria to drive blindly down a familiar road, completely abstracted as if a guarding angel is looking upon us. Therefore lack of practice might affect this automaticity (Rostami and Ashayeri 2009). Hence, we presume that post lockdown in a small group of the population, the RT falls beyond the constant range, with a growing probability of misjudgments and collisions. To forestall this, the driver has to make some voluntary adjustments with either the speed or the distance.

\subsection{Modulation of which of the above two aspects of driving would yield better safety on the road?}

Recalling our driving lessons, do you remember learning to speed up at the very beginning? Or simply mastering the basics first, which included driving slowly at a safe distance from the other vehicles. Likewise, downscaling the speed would decrease the impact during a collision by abating the momentum. Once proficiency is achieved, we acquire the skill of driving faster and maintaining much less distance from the other vehicles. Therefore, a need for detailed studies should be carried out to understand the activity of the brain during and after the driving lessons and how and when it switches from one to another to manipulate speed and distance. We also propose ascertaining a specific centre in the brain that controls the speed. Adhering to our primitive learning behavior where distance was given more importance than speed, we would keep a safe distance. 
Hence as explained above, maintaining a safe distance at the lowest speed would be the safest approach, coexisting with uniform speed calculations. We have used it to sensitize the public to quantify their speed post lockdown as patience is something you admire in the driver behind you, but not in the one ahead. The theme of the study was to heave the quote 'speed thrills, but it kills'.

So far, by reducing speed, we can say that we have succeeded in reducing a collision's impact. Collisions still occur at a slower speed as well. So, it is the quickness of action which can reduce the probability of a collision. Therefore, there is a need for a parameter that can measure this quickness. Visual RT can be considered as an index for this quickness (Western and Long 1996). This can also be used to express it in another way.

\subsection{Alternate Facet For RT}

Imagine our brain as a central processing unit (CPU) that works at a particular speed, arbitrarily at $8 \mathrm{GHz}$. The driving program that has been installed through driving lessons is compatible with this speed of the processor. Our nervous system is a unique computer that can upgrade itself to a higher speed with use and vice versa. Post lockdown, the average driving speed has decreased, which can be attributed to our assumption of slowing in RT. During this period, the brain tries to run the same driving program, but the speed is slower (say $6 \mathrm{GHz}$ ), as extrapolated from the present study results. Therefore, as with a CPU in which there are chances of incompatibility or loss of function, even here, there are chances of a crash. Despite having no idea about the rate of up-grading Brain-CPU, we can reprogram our program for driving to make it compatible with the CPU's present speed.

In our study, we can derive the processing speed as follow-

Total active participation time $=10(S R T+L R T)$

Frequency $=20 / 10(S R T+L R T)=2 /(S R T+L R T)$

Our study example shows if the SRT and LRT of a person are $0.5 \mathrm{~s}$ and $0.7 \mathrm{~s}$, respectively, then considering average RT, the average frequency of his brain processor $=1.67 \mathrm{~Hz}$ (or in a range of $1.43 \mathrm{~Hz}$ to $2 \mathrm{~Hz}$ ). The actual processing speed would be much better than this if we considered the complex analysis by taking the neurons and their synapses as an individual biological CPU, which runs parallel (Nagarajan and Stevens 2008). However, in this case, we have considered the simplified analysis by taking a group of neurons in a specific brain area as a single CPU, thus focusing on gross problems. As akin to the experimentation of task switching by N. Sinha et al., we present an interesting parameter that can be derived is the rate of change of frequency (ROCOF) by the brain, i.e., the rate at which processor shifts either slow to fast or reverse of it (Sinha et al. 2006).

As the speed of the processor increases, the person speeds up. But the incident of a crash depends on how efficiently the brain deals with the transition between different events. So, on-road, the ROCOF for slow to fast and fast to slow is important for controlling the vehicle. Hence the compatibility of a driver is 
not only based on the RT for a particular event but also on the rapidity to switch from one event to another, which can be evident as 'switch cost' in the experimental model of D.W. Schneider et al. (Schneider and Logan 2005).

\subsection{Shortcomings of our study}

Since the difficulty level of the visual stimulus test page was of very easy/low level and the number of clicks that provide the response time was limited to just 20, a very crude result was obtained. In different studies, after excluding scientific errors, the statistically normal RT is $310 \mathrm{~ms}$ [4], while in our study, the technical errors have not been taken into consideration. Therefore, the present study cannot standardize the RT and cannot be implemented on a large scale.

\subsection{The Implication For Future Research}

Driving is a multifactorial skill, and visual RT is a part of it. Also, the data is very crude without considering scientific error. Therefore, this study's results, based on visual RT, cannot have a generalized implementation. However, it is enough to prime the public to 'Slowdown Post Lockdown' and drive consciously to prevent mishaps.

For the above reason, we require actual performance data in different conditions by different classes of the population on a simulator having tests ranging from simple to complex levels. This performance data can provide comprehensive results by analyzing the events where the prompt response is required and the events where no response is required.

\subsubsection{Suggested simulator}

Experiments conducted by C. Ariën et al. and A. Akbari et al. suggest that though the traditional approach of obtaining real-time data of driving on the road is irreplaceable, it has many constraints which can be overcome by using surrogate techniques like a simulator that is close to various traffic environments and can provide with eventful data (Akbari and Haghighi 2020; Ariën et al. 2013). The current period marks the dawn of technological evolution where 'mixed reality' has come into bloom, giving us an up-close experience of the new technology. Such immersive technology can be used to design a near-perfect driving simulation. The 4D simulation rides, VR gears, 12D technology (by Voyage Technologies), spatial computer (by Magic leap), and Google glass (by Google) are just a sneak peek into the colossal orb of this technology. Studies like the one focusing on humans like an advanced driver assistance system or the one that involves psychophysical microsimulation models like the Aimsun and VISSIM (Astarita et al. 2019) form a base for more futuristic ventures. A simulator with the capability to analyze various RT components, and the factors affecting them, can yield valuable data. The task so designed for the simulator should have various levels of difficulties that can be made to complete in a stipulated time or at will, simultaneously measuring the rate of occurrence of failure or mistakes. 


\subsubsection{Graphics of simulator}

The simulator's graphics should be designed in the backdrop of the participants' city overlaid by different terrains. It can be tailor-made for factors relating to the environment, particularly lighting, road surface, and weather conditions.

\subsubsection{Calibration}

A system is said to be well-calibrated in the timing and the threshold of the stimuli if it coincides with the participants' perception. This estimation helps avoid inaccurate determination of the start time and stop time of stimuli and their response. Also, incorporating maximum modalities of stimuli for calibration and testing would produce a near driving experience. Paradigmatically for a visual stimulus, timing and threshold should be standardized as per the individual's field of vision by some simplified test like the confrontation test.

\subsubsection{Controls and sensors}

The response to any stimuli is in the form of either maneuvering of steering, brake, or pedal. Therefore, any movement in either must be considered for RT. For instance, if in a condition that would require maneuvering the steering as an essential move rather than braking, then relying on braking to derive RT would be erroneous. It can be exemplified as a condition where braking could be an obvious choice. If a rider averts a conflict by maneuvering the steering or grip instead of braking, it should be preferentially counted as the initial response. Therefore, the system should be able to sense a permissible movement of either to calculate the RT. Apart from the vehicular speed, the system should sense the variance in time and distance concerning trivial changes in acceleration or braking, or steering.

\subsubsection{Analysis}

As expertise lies in diagnosing based on the pattern of symptoms, and their timely detection can forecast the onset of disease and its remedial measure. Similarly, an eye-tracking metrics system instigates us to propose that the system should be able to determine the speed of the brain using RT and compute ROCOF. Through this computation, the system can delineate the rate at which the change occurs (Merat et al. 2014). Considering it with an EEG illustration, which gives us the changes in a wave pattern from one activity to another, it does not give one waveform's rapidity to another. If the brain's speed is measured frequently from initiation until the end, then the results computed from the average speed and ROCOF would be more precise. ROCOF, though crude, helps in detecting this rate of change easily. Hence its application for predicting the rate would be a great accomplishment.

The simulation test analysis should be concluded by a detailed report depicting the speed attained, the number of mistakes, time taken to complete, safe distance, acceleration/deceleration used, and ROCOF as per different conditions and terrain. Thus, taking all this into account, the report is to be followed by a remark for suggested driving speed, safe distance, and acceleration/deceleration concerning different conditions and terrains. 
By doing so, a range of critical processing speed and ROCOF for the different age groups can be determined for driving in easy or tough terrain, and a person's best performance at a particular speed limit can also be obtained. A participant's driving skill in different simulation test courses can be classified as in Table 3.

Table 3. Classification of Drivers.

\begin{tabular}{|llllll|}
\hline Category & $\begin{array}{l}\text { Speed of Brain } \\
\text { processor } \mathrm{F}_{\mathrm{c}}(\mathrm{Hz})\end{array}$ & ROCOF $_{\mathrm{c}}$ & $\begin{array}{l}\text { Slow driving } \\
\text { compatibility }\end{array}$ & $\begin{array}{l}\text { Fast driving } \\
\text { tendency }\end{array}$ & $\begin{array}{l}\text { Probability of } \\
\text { Collision }\end{array}$ \\
\hline 1 & - & +++ & +++ & - \\
\hline 2 & - & - & +++ & + & + \\
\hline 3 & & - & - & ++ & ++ \\
\hline 4 & - & - & - & +++ \\
\hline
\end{tabular}

c critical value, increase above or ${ }^{-}$decrease below c, +++ high, ++moderate, +low, - least.

It shows the percentage of the population falling in various categories. Based on the analysis of the tabular data, traffic rules can be formulated and stringently followed. The standardized range of the above parameters can also be used as a marker for fitness in a certain profession like defense skills, aviation, transport, and deriving a provisional license. Not much has been studied about the corelationship between duration of non-activity and duration required to attain the best RT in that activity, especially driving. Therefore, this type of stratification can help the researcher classify drivers, which requires further investigation to answer.

\subsection{Conflicting Views And Its Justification Regarding The Study}

Many would logically assert that fast drivers and slow drivers can nullify their effects post lockdown, i.e., the euphoric like the juveniles can try to over speed and elderly will intuitively tend to slow down. Moreover, considering that the traffic rules and regulations are not followed stringently in developing countries, the study's generalized application seems difficult and erroneous. We concur with the given logic, but the percentage of fast and slow drivers cannot be derived by any means. Also, there would be interference in the brain due to a lack of driving. The RT or reflex action for other factors that play a role in driving would also be slowed down as the brain's specific areas would not have undergone plasticity to deal with beyond the range of accustomed RT, ultimately leading to the collision. Therefore any technique that can reduce the impact or number of collisions will be benevolent to society. As they say, benevolence is absolute, and real kindness costs nothing but means everything.

\section{Conclusion}


The present study was designed under the speculation of a post lockdown scenario. Where we succeeded in designing and estimating post lockdown speed based on visual RT using a website. Our supposition of utter chaos on the roads due to variation in reaction time holds by our findings, which correlate the reaction time with the days of lockdown. Therefore, visual RT can be appropriate in controlling vehicle speeds.

\section{Abbreviations}

$\mathrm{RT}=$ Reaction time

SRT $=$ Shortest reaction time

$\mathrm{LRT}=$ Longest reaction time

aSRT $=$ Average shortest reaction time

aLRT = Average longest reaction time

ASALD $=$ Average Speed after Lock down

ACS $=$ Average Comfortable Speed

$\mathrm{CPU}=$ Central processing unit

ROCOF $=$ Rate of change of frequency

\section{Declarations}

Ethics approval and consent to participate - Not Applicable

Consent for publication - Not Applicable

Availability of data and materials - The datasets used and/or analysed during the current study are available from the corresponding author on reasonable request.

Competing interests - The authors declare that they have no competing interests.

Funding - This research did not receive any specific grant from funding agencies in the public, commercial, or not-for-profit sectors.

\section{Acknowledgements}

Our profound gratitude to the CEO of 'Kinetic Dreamers,' Mr. Bhavesh Rajdeep, for accelerating our idea to reality by endeavoring in the website's thoughtful design. Chaperoning in the last lap of our expedition by their valuable and constructive suggestions and useful critiques our exceptional prepublication reviewers 
Dr. Kalpita Shringarpure (M.D. P.S.M, M.S. University), Dr. Vismay Trivedi (PhD. in Applied physics, Fellowship at Bar Ilan University, Israel), Dr. Samir Bhagora (M.D. Physiology, Section Officer; Revenue Dept, Gandhinagar) and Dr. R.K.Patel (M.D. Physiology, M.S. University) have ostensibly reviewed our work deserving our deepest appreciation. We extend our acknowledgment to Amanda D. (Ph.D.) of 'Essaypro.com' for her language editing expertise.

\section{References}

1. Akbari A, Haghighi F. Traffic calming measures: An evaluation of four low-cost TCMs' effect on driving speed and lateral distance. IATSS Res. 2020;44(1).

2. Ariën C, Jongen EMM, Brijs K, Brijs T, Daniels S, Wets G. A simulator study on the impact of traffic calming measures in urban areas on driving behavior and workload. Accid. Anal. Prev. 2013;61.

3. Astarita V, Festa DC, Giofrè VP, Guido G. Surrogate Safety Measures from Traffic Simulation Models a Comparison of different Models for Intersection Safety Evaluation. Transp. Res. Procedia. 2019.

4. Bokare PS, Maurya AK. Acceleration-Deceleration Behaviour of Various Vehicle Types. Transp. Res. Procedia. 2017.

5. Calhoun VD, Pekar JJ, McGinty VB, Adali T, Watson TD, Pearlson GD. Different activation dynamics in multiple neural systems during simulated driving. Hum. Brain Mapp. 2002;16(3).

6. Collins LF, Long CJ. Visual reaction time and its relationship to neuropsychological test performance. Arch. Clin. Neuropsychol. 1996;11(7).

7. Huang J, Yan E, Cheung G, Nagappan N, Zimmermann T. Master Maker: Understanding Gaming Skill Through Practice and Habit From Gameplay Behavior. Top. Cogn. Sci. 2017;9(2).

8. Li Y, Chen Y. Driver vision based perception-response time prediction and assistance model on mountain highway curve. Int. J. Environ. Res. Public Health. 2017;14(1).

9. Merat N, Jamson AH, Lai FCH, Daly M, Carsten OMJ. Transition to manual: Driver behaviour when resuming control from a highly automated vehicle. Transp. Res. Part F Traffic Psychol. Behav. 2014;27(PB).

10. Nagarajan N, Stevens CF. How does the speed of thought compare for brains and digital computers? Curr. Biol. 2008.

11. Olson PL, Sivak M. Perception-response time to unexpected roadway hazards. Hum. Factors. 1986;28(1).

12. Rostami HR, Ashayeri H. Effects of motor skill practice on reaction time and learning retention in Parkinson's disease. Neurol. India. 2009;57(6).

13. Sakai H, Ando T, Sadato N, Uchiyama Y. Speed-related activation in the mesolimbic dopamine system during the observation of driver-view videos. Sci. Rep. 2018;8(1).

14. Schneider DW, Logan GD. Modeling task switching without switching tasks: A short-term priming account of explicitly cued performance. J. Exp. Psychol. Gen. 2005. 
15. Sinha N, Brown JTG, Carpenter RHS. Task switching as a two-stage decision process. J. Neurophysiol. 2006;95(5).

16. W.H.O. Global Status Report on Road Safety 2018: Summary. World Heal. Organ. 2018.

17. Western SL, Long CJ. Relationship between reaction time and neuropsychological test performance. Arch. Clin. Neuropsychol. 1996;11(7).

18. Woods DL, Wyma JM, William Yund E, Herron TJ, Reed B. Age-related slowing of response selection and production in a visual choice reaction time task. Front. Hum. Neurosci. 2015;9(APR).

19. Zhu X, Hu X, Chiu YC. Design of Driving Behavior Pattern Measurements Using Smartphone Global Positioning System Data. Int. J. Transp. Sci. Technol. 2013;2(4). 